\title{
The role of perceptual expectation on repetition suppression: a quest to dissect the differential contribution of probability of occurrence and event predictability
}

\author{
Elia Valentini ${ }^{1,2 *}$ \\ 1 Dipartimento di Psicologia, Sapienza Università di Roma, Roma, Italy \\ 2 Istituto di Ricovero e Cura a Carattere Scientifico, Fondazione Santa Lucia, Roma, Italy \\ *Correspondence: elia.valentini@uniroma1.it
}

\section{A commentary on}

Human scalp electroencephalography reveals that repetition suppression varies with expectation

by Summerfield, C., Wyart, V., Johnen, V. M., and de Gardelle, V. (2011). Front. Hum. Neurosci. 5:67. doi: 10.3389/ fnhum.2011.00067

Stimulus repetition is associated with an automatic reduction of cortical activity (e.g., Walter et al., 1964). This phenomenon, often termed repetition suppression (RS), has been reported at multiple spatial scales (Grill-Spector et al., 2006 for a review), from the level of individual cortical neurons in monkeys (e.g., Carandini and Ferster, 1997) to the level of electrical and hemodynamic changes in humans (e.g., Summerfield et al., 2008; Garrido et al., 2009). Traditionally, RS has been characterized either in terms of a process of habituation (Fruhstorfer et al., 1970; Fruhstorfer, 1971), such as that defined by the orienting response theory (Sokolov, 1963), or in terms of a process involving the refractory period of neural generators (Ritter et al., 1968; Budd et al., 1998). Such opposite interpretation is reminiscent of two main theoretical views on sensory representation: a top-down building of perceptual expectations from fronto-parietal cortices (Henson, 2003; Friston, 2005) and a bottom-up automatic tuning (e.g., sharpening mechanism) of perceptual information through sensory cortices (Desimone, 1996; Wiggs and Martin, 1998), respectively.

Importantly, a recent study attempting to replicate face-repetition effects on BOLD signal in the extrastriate visual cortex (Summerfield et al., 2008) reported no effect of RS on single inferotemporal neurons (Kaliukhovich and Vogels, 2011). This null finding provides indirect support to the idea that the effects of repetition would be largely associated to neural adaptation mechanisms rather than to "perceptual expectation." However, in a more recent fMRI study Larsson and Smith (2011) replicated Summerfield et al.'s (2008) and showed that when attention was diverted away from the stimuli the effects of stimulus expectation disappeared but a significant RS was still evident. Thus, it is entirely possible that RS, as indexed by fMRI adaptation paradigms, may reflect a combination of bottom-up automatic tuning of neuronal excitation and top-down building of perceptual expectation.

In a recent issue of Frontiers in Human Neuroscience Summerfield et al. (2011) tackled this challenging issue by testing RS effects on the human electroencephalogram (EEG) signal. They used a $2 \times 2 \times 2$ factorial design comparing stimulus repetition (repetition vs. alternation) with expectation (expect repetition vs. expect alternation) and volatility (volatile vs. stable). Essentially derived from their previous study (Summerfield et al., 2008), this design had a new important exception: trials sequences were built in such a fashion that the probability of a repetition vs. an alternation changed between 0.2 and 0.8 every 10 trials (volatile condition) or 30-40 trials (stable condition).

By changing the probability of occurrence these authors aimed to test the hypothesis that the repetition $\times$ expectation interaction should have appeared during stable periods rather than during volatile periods. Crucially, to test this hypothesis an original analytic approach was employed whereby a sequential Bayesian learner computed single-trial maximum likelihood estimates of the expected probability of repetition $p$ (rep) and the volatility $v$ for each subject, and subsequently used these single-trial estimates as regressors for EEG single-trial analyses. Aside from this innovative analytic method,
Summerfield et al. (2011) provided also a thorough analysis of both phase- and nonphase-locked EEG responses by computing both standard averaging of time-locked deflections and time-frequency decomposition of EEG oscillatory activity.

In sum, repetition effects were observed at parietal electrodes and at central electrodes between $\sim 300$ and 400 ms poststimulus. In particular, at central electrodes (CP3, CP4, C3, C4, CPz, Cz) an interesting two-way repetition $\times$ expectation interaction was detected by means of the Bayesian regression. This effect was accounted for by greater RS when repetitions were expected than when they were unexpected. Interestingly, the analysis of spectral power in the theta-band range (between 200 and $700 \mathrm{~ms}$ ) was sensitive to the three-way interaction between repetition, expectation, and volatility: power was reduced during repetitions compared to alternations but only when repetitions were expected, and only when the probability of occurrence was stable. Therefore, expectation reliably modulated RS only during stable periods whereas no reliable modulation by expectation was observed during volatile periods.

Such finding has important implications for all the other electrophysiological studies of RS in other sensory modalities. For instance, recent studies on cortical responses elicited by repeated nociceptive stimuli of identical energy (Iannetti et al., 2008; Wang et al., 2010), showed that RS can be clearly observed in event related potentials (ERPs) evoked by nociceptive laser stimuli as well as observed in the auditory modality (e.g., Rosburg, 2004; Viswanathan and Jansen, 2010). In addition, it has been suggested that this phenomenon is strongly determined by the novelty (as function of saliency) of the eliciting stimulus (Wang et al., 2010; Valentini et al., 2011), rather than by neural refractoriness (Truini et al., 
2004, 2007). This view is in agreement with Summerfield et al.'s $(2008,2011)$ interpretation of their hemodynamic and electrophysiological findings. In addition, however, Valentini et al. (2011) suggested that the suppression of the EEG signal magnitude can be mainly explained by a lack of change (or deviancy - as function of novelty) in the sensory information contained in the repeated stimulus and less impacted by the fact that the change of incoming sensory information is unexpected.

Crucially, the ERPs literature seems to implicitly confer the same meaning to the two constructs of novelty and uncertainty: the occurrence of an unpredictable deviant event in the environment. However, as elegantly emphasized by Parmentier et al. (2011): novel sensory stimuli "are not only rare but also unexpected. Probability and predictability are often used interchangeably in oddball studies but these concepts are not synonymous." In other words, expected rare events may not trigger the same amount of neural activation as that elicited by unexpected rare events. Similarly, the cortical modulation exerted by a change of a sensory feature (e.g., modality change) cannot be conceived as always representing novelty, as it is possible to have a change without it being novel.

In conclusion, Summerfield et al.'s (2011) study is an elegant first attempt to disentangle these conceptual and operational issues. Future research may focus on how and when both bottom-up and top-down variables differentially contribute to shortterm habituation processes, and what are their neurophysiological underpinnings.

\section{ACKNOWLEDGMENT}

The author acknowledges the support of the British Academy, Small Research Grant award scheme.

\section{REFERENCES}

Budd, T. W., Barry, R. J., Gordon, E., Rennie, C., and Michie, P. T. (1998). Decrement of the N1 auditory event-related potential with stimulus repetition: habituation vs. refractoriness. Int. J. Psychophysiol. 31, 51-68.

Carandini, M., and Ferster, D. (1997). A tonic hyperpolarization underlying contrast adaptation in cat visual cortex. Science 276, 949-952.

Desimone, R. (1996). Neural mechanisms for visual memory and their role in attention. Proc. Natl. Acad. Sci. U.S.A. 93, 13494-13499.

Friston, K. (2005). A theory of cortical responses. Philos. Trans. R. Soc. Lond. B Biol. Sci. 360, 815-836.

Fruhstorfer, H. (1971). Habituation and dishabituation of the human vertex response. Electroencephalogr. Clin. Neurophysiol. 30, 306-312.

Fruhstorfer, H., Soveri, P., and Jarvilehto, T. (1970). Short-term habituation of the auditory evoked response in man. Electroencephalogr. Clin. Neurophysiol. 28, 153-161.

Garrido, M. I., Kilner, J. M., Kiebel, S. J., Stephan, K. E., Baldeweg, T., and Friston, K. J. (2009). Repetition suppression and plasticity in the human brain. Neuroimage 48, 269-279.

Grill-Spector, K., Henson, R., and Martin, A. (2006) Repetition and the brain: neural models of stimulus-specific effects. Trends Cogn. Sci. (Regul. Ed.) 10, 14-23.

Henson, R. (2003). Neuroimaging studies of priming. Prog. Neurobiol. 70, 53-81.

Iannetti, G. D., Hughes, N. P., Lee, M. C., and Mouraux, A. (2008). Determinants of laser-evoked EEG responses: pain perception or stimulus saliency? J. Neurophysiol. 100, 815-828.

Kaliukhovich, D. A., and Vogels, R. (2011). Stimulus repetition probability does not affect repetition suppression in macaque inferior temporal cortex. Cereb. Cortex 21, 1547-1558.

Larsson, J., and Smith, A. T. (2011). fMRI Repetition suppression: neuronal adaptation or stimulus expectation? Cereb. Cortex. doi: 10.1093/cercor/bhr119

Parmentier, F. B., Elsley, J. V., Andres, P., and Barcelo, F. (2011). Why are auditory novels distracting? Contrasting the roles of novelty, violation of expectation and stimulus change. Cognition 119, 374-380.

Ritter, W., Vaughan, H. G. J., and Costa, L. D. (1968). Orienting and habituation to auditory stimuli: a study of short-term changes in averaged evoked responses. Electroencephalogr. Clin. Neurophysiol. 25, 550-556.

Rosburg, T. (2004). Effects of tone repetition on auditory evoked neuromagnetic fields. Clin. Neurophysiol. $115,898-905$.
Sokolov, E. N. (1963). Higher nervous functions; the orienting reflex. Annu. Rev. Physiol. 25, 545-580.

Summerfield, C., Trittschuh, E.H., Monti, J.M., Mesulam, M. M., and Egner, T. (2008). Neural repetition suppression reflects fulfilled perceptual expectations. Nat. Neurosci. 11, 1004-1006.

Summerfield, C., Wyart, V., Johnen, V. M., and de Gardelle, V. (2011). Human scalp electroencephalography reveals that repetition suppression varies with expectation. Front. Hum. Neurosci. 5:67. doi: 10.3389/ fnhum.2011.00067

Truini, A., Galeotti, F., Cruccu, G., and Garcia-Larrea, L. (2007). Inhibition of cortical responses to Adelta inputs by a preceding C-related response: testing the "first come, first served" hypothesis of cortical laser evoked potentials. Pain 131, 341-347.

Truini, A., Rossi, P., Galeotti, F., Romaniello, A., Virtuoso, M., De Lena, C., Leandri, M., and Cruccu, G. (2004) Excitability of the Adelta nociceptive pathways as assessed by the recovery cycle of laser evoked potentials in humans. Exp. Brain Res. 155, 120-123.

Valentini, E., Torta, D. M., Mouraux, A., and Iannetti, G. D. (2011).Dishabituation of laser-evoked EEG responses: dissecting the effect of certain and uncertain changes in stimulus modality. J. Cogn. Neurosci. 23, 2822-2837.

Viswanathan, D., and Jansen, B. H. (2010). The effect of stimulus expectancy on dishabituation of auditory evoked potentials. Int. J. Psychophysiol. 78, 251-256.

Walter, W. G., Cooper, R., Aldridge, V. J., McCallum, W. C., and Winter, A. L. (1964). Contingent negative variation: an electric sign of sensorimotor association and expectancy in the human brain. Nature 203, 380-384.

Wang, A. L., Mouraux, A., Liang, M., and Iannetti, G. D. (2010). Stimulus novelty, and not neural refractoriness, explains the repetition suppression of laserevoked potentials. J. Neurophysiol. 104, 2116-2124.

Wiggs, C. L., and Martin, A. (1998). Properties and mechanisms of perceptual priming. Curr. Opin. Neurobiol. 8, 227-233.

Received: 24 October 2011; accepted: 02 November 2011; published online: 21 November 2011.

Citation: Valentini E (2011) The role of perceptual expectation on repetition suppression: a quest to dissect the differential contribution of probability of occurrence and event predictability. Front. Hum. Neurosci. 5:143. doi: 10.3389/ fnhum.2011.00143

Copyright (C) 2011 Valentini. This is an open-access article subject to a non-exclusive license between the authors and Frontiers Media SA, which permits use, distribution and reproduction in other forums, provided the original authors and source are credited and other Frontiers conditions are complied with. 